\title{
Scratching the Bose surface
}

\author{
Subir Sachdev用 \\ Department of Physics, Yale University, \\ P.O. Box 208120, New Haven CT 06520-8120
}

\begin{abstract}
This is a 'News and Views' article (Nature 418, 739 (2002)) discussing recent proposals for ground states of many boson systems which are neither superfluids nor Mott insulators.
\end{abstract}


There are two distinct types of particles in nature: fermions and bosons. But it seems bosons may assume similar characteristics to fermion systems in the low-temperature regime typical of Bose-Einstein condensation.

Particles known as fermions (such as electrons) obey the Pauli exclusion principle, which decrees that only a single fermion can occupy a particular state, such as an orbital in an atom. But the second family of particles, bosons (such as helium atoms), have no corresponding restrictions on their occupation of states. This distinction in their individual properties also carries over to the collective properties of large numbers of fermions or bosons at low temperatures. The typical quantum state of many fermions is a Fermi liquid, formed, for example, by the valence electrons in all metals: the electrons can move from atom to atom in this state and conduct electricity across macroscopic distances, albeit with a finite resistance. In contrast, bosons typically form a Bose-Einstein condensate, which is the basis of superfluidity in helium and its ability to flow perpetually with negligible dissipation.

Now, Paramekanti and colleagues [1], in an article to appear in Physical Review B, have proposed an innovative state for boson systems which shares many of its characteristics with the metallic state normally associated with fermions. According to Paramekanti et $a l$. , strong interactions among the bosons entangle them in a delicate quantum-mechanical superposition that mimics the properties of fermions.

One of the defining properties of a Fermi liquid is its Fermi surface. In a metal, we can label the states available to the fermionic electron by its momentum vector $\mathbf{k}$. The Fermi surface resides in $\mathbf{k}$-space, and in the lowest-energy state of the metal, electrons occupy all states inside the Fermi surface, while those outside the surface remain unoccupied (Fig. 1a). This surface is of particular physical importance because it defines the low-energy excitations observed experimentally: a fluctuation in the density of the electrons is created by moving an electron from an occupied state inside the Fermi surface to an unoccupied state outside the Fermi surface; probes that eject an electron from the metal (such as an energetic photon in a photoemission experiment) will act most efficiently on states just inside the Fermi surface. The Pauli exclusion principle, by enforcing single occupancy of fermionic states, is crucial in defining the concept of a Fermi surface, as electrons can only be removed (or added) from states inside (or outside) the Fermi surface.

The striking proposal of Paramekanti et al. is that bosons can also form a Bose liquid with a Bose surface, analogous to the Fermi liquid and Fermi surface. Clearly, as there is 
no Pauli exclusion principle for bosons, the Bose surface cannot be the boundary between occupied and unoccupied states. Instead, the Bose surface defines the locus of points in momentum space with low-energy excitations (Fig. 1b). Nevertheless, the excited states of the Bose liquid are defined by the Bose surface in a manner that is very reminiscent of the Fermi surface in a Fermi liquid: low-energy fluctuations in the density of the bosons involve rearrangement of the states near the Bose surface, as does an excitation that ejects a boson from the liquid.

Paramekanti et al. also discuss the conditions under which this fascinating Bose liquid may form. Weakly interacting bosons invariably collapse into the single state of a BoseEinstein condensate, but merely turning up the strength of the repulsive interactions between the bosons is not enough to establish a Bose liquid. In fact, this leads to a state known as a Mott insulator, in which the bosons localize in a regular, crystalline arrangement. The transition between the Bose-Einstein condensate and the Mott insulator in a trapped gas of rubidium atoms was observed recently in a beautiful experiment by Greiner et al. [2].

To deter the bosons from forming a Bose-Einstein condensate or a Mott insulator, a more complex interaction between the bosons is necessary. In particular, a large contribution from 'boson ring exchanges' appears to be crucial. This feature arises because the quantummechanical description - or wavefunction - of the ground state contains a superposition of states in which pairs of bosons move in a correlated manner around a ring. In such a state, the positions of the bosons around the ring are uncertain, but a measurement that locates one boson at a particular site also specifies the position of the second boson at another site on the ring.

Stimulated by the proposal of Paramekanti et al., Sandvik et al. [3] have already reported results from a computer simulation of the simplest quantum description of boson behaviour in two dimensions, including a large boson-ring-exchange term. So far, they have not found a state with a Bose surface, but have instead obtained a new form of Mott insulator in which the bosons crystallize on half the horizontal bonds of the lattice; this type of state had also been predicted [4].

Paramekanti et al. [1] also discuss the prospects for experimental discovery of their Bose liquid state. They suggest that such a state might be formed by pairs of electrons (which act like composite bosons and are known as Cooper pairs) in the cuprate compounds that superconduct at high temperatures. Mason and Kapitulnik [5 have recently reported an 
unexpected regime of metallic conduction in a disordered thin film of $\mathrm{Mo}_{43} \mathrm{Ge}_{57}$ in a magnetic field - the film is a superconductor in zero field, and a Bose liquid of Cooper pairs is an intriguing possibility for the metallic phase. And there are other competing theories [6, 7, 8], involving a more fundamental role for disorder in the film. It is clear that the resolution of the puzzle set by Paramekanti et al. brings the prospect of much exciting new physics.

* Electronic address: subir.sachdev@yale.edu; URL: http://pantheon.yale.edu/ ${ }^{\text {subir }}$

[1] Paramekanti, A., Balents, L. \& Fisher, M. P. A. cond-mat/0203171.

[2] Greiner, M., Mandel, O., Esslinger, T., Hansch, T. W. \& Bloch, I. Nature 415, 39 (2002).

[3] Sandvik, A. W., Daul, S., Singh, R. R. P. \& Scalapino, D. J. cond-mat/0205270.

[4] Park, K. \& Sachdev, S. Phys. Rev. B 65, 220405 (2002).

[5] Mason, N. \& Kapitulnik, A. Phys. Rev. B 64, 060504 (2001).

[6] Spivak, B., Zyuzin, A. \& Hruska, M. Phys. Rev. B 64, 132502 (2001).

[7] Dalidovich, D. \& Phillips, P. Phys. Rev. Lett. 89, 027001 (2002).

[8] Das, D. \& Doniach, S. Phys. Rev. B 64, 134511 (2001). 


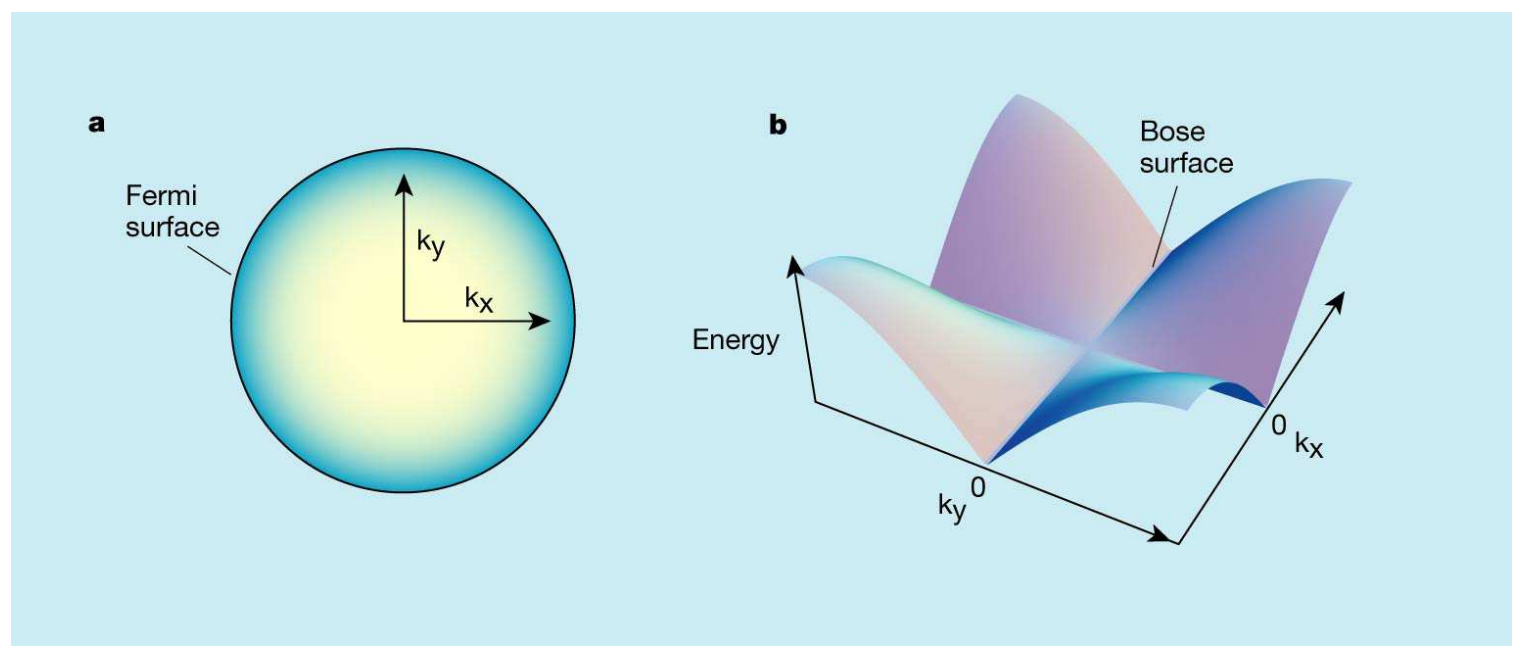

FIG. 1: Fermi and Bose surfaces in two dimensions. (a) Fermionic electrons in a metal obey Pauli's exclusion principle - there can be only one fermion in each quantum state. The Fermi surface marks the divide, defined by the particles' momentum components $k_{x}$ and $k_{y}$, between occupied and unoccupied momentum states. (b) Although bosons do not obey the exclusion principle, Paramekanti et al. [1] propose that they can form a Bose surface in a Bose liquid, analogous to the Fermi surface for fermions. In the spectrum of boson excitations in the Bose liquid, the excitation energy (vertical axis) vanishes near the Bose surface (which follows the lines $k_{x}=0$ and $k_{y}=0$ ): a Fermi liquid has a similar spectrum of excitations that vanishes on its circular Fermi surface. 